\author{
Military Technical College \\ Kobry El-Kobbah, \\ Cairo, Egypt.
}

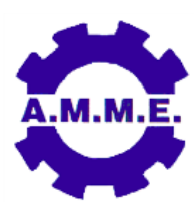

$17^{\text {th }}$ International Conference on Applied Mechanics and Mechanical Engineering.

\title{
MODAL ANALYSIS AND TESTING OF MODIFIED RE-ENTRANT HEXAGONAL HONEYCOMB CORES
}

\author{
K. M. Abozied ${ }^{1}$, M. O. Osman² and T. H. Elmahdy ${ }^{3}$
}

\begin{abstract}
In the present work, the vibrational behaviors of cellular auxetic honeycomb cores were investigated. Classical hexagonal re-entrant honeycomb structure and two newly developed designs namely, splined and stiffened re-entrant honeycomb structures were used in this investigation. The newly developed structures were found to have in-plane negative Poisson's ratios with in-plane stiffness higher than that of classical re-entrant hexagonal structure. Finite elements models were developed for the three structures to obtain the mechanical properties and the natural frequencies. The finite elements results showed that the honeycombs exhibit different modes of vibrations namely, bending, torsional and axial modes. It was found that the natural frequencies in bending decreases with increase of the rib length. This behavior was directly linked to the increase of the in-lane relative density and stiffness of the honeycomb. Experiments on the honeycomb cores of the three structures were carried out to obtain the cores natural frequencies and their respective mode shapes. The finite element results were verified with the experimental results for various geometrical parameters and good agreement between the results was obtained.
\end{abstract}

\section{KEY WORDS}

Honeycombs, Auxetic, Negative Poisson's ratio, In-plane properties, Re-entrant, Vibrational characteristics, Natural frequency. 


\section{NOMENCLATURE}

$\begin{array}{cl}\text { Symbol } & \text { Meaning } \\ b & \text { The auxetic structure depth } \\ E_{s} & \text { Young's modulus of the solid material of the structure } \\ E_{x} & \text { Young's modulus in x-direction of the auxetic network } \\ s_{y} & \text { Young's modulus in x-direction of the auxetic network } \\ h & \text { Ligament height for unequal rib re-enterent hexagonal cell } \\ l & \text { Ligament length } \\ t & \text { rib thickness } \\ \theta & \text { The rib orientation } \\ v_{x y}, v_{y x} & \text { In-plane Poisson's ratio of the tructure }\end{array}$

\section{INTRODUCTION}

There is a growing interest in petroleum, construction, automotive, aerospace industries to use new materials to reduce weight of systems components for better performance, reducing fuel consumption, exhaust emission and noise reduction. Advanced materials such as honeycomb structures come to light as a feasible candidate in these fields. This type of structures can be used in cabs, floors, walls, doors, panels and roofs for vans, trucks, trailers trains and airplanes. The honeycombs and their sandwich panels find interest also in state-of the-art new buildings as noise and thermal insulation members. The selected area of research presented in this paper is focusing on the development of honeycomb cores for sandwich structures used in the above mentioned applications especially in places where lightweight, high toughness, vibration and acoustics damping are required. Moreover, the use of this type of structures finds the same interest in many other application areas such as ships and yacht industry and it represents state-of-the-art in materials technology [1].

Honeycomb cores that have in-plane negative Poisson's ratio (auxetic) are recently developed with different shapes and geometries [2-9]. An example of how to achieve auxetic behavior is through modification of the geometry of hexagonal honeycomb unitcell into one in which the hexagonal cells adopt a re-entrant hexagonal shape as shown in Table 1. Re-entrant hexagonal honeycomb is auxetic when the deformation mechanism is occurred by flexing or hinging of the cell ligaments [3-5]. The advantages of the auxetic honeycombs over the traditional hexagonal positive poison's ratio honeycombs are the enhancements of many physical properties such as energy absorption capability, plane strain fracture toughness and the ability to form synclastic (dome shape) curvatures under out-of-plane bending [3, 9]. Auxetic honeycombs have recently attracted many researchers to develop new designs that can be implemented in many industrial applications for their superior properties over the conventional ones. [3-9]. Auxetic honeycomb structures have also been good candidate for the design of panels for several applications (10-11] and for morphing wings [13-15] and many other aerospace structures [15-18].

In the present work, the vibrational characteristics of the hexagonal re-entrant honeycomb structure and their modified designs were studied. It has been shown that for the re-entrant configuration of the hexagonal honeycomb, the behaviour of 
the honeycomb exhibits the auxetic behaviour i.e. the resulting in-plane Poisson's ratio is negative. Moreover, the in-plane modulus of elasticity is reduced significantly [19], which resulted in very low natural frequencies. The study also conducted on the two new improved stiffness designs of the unitcell based on the basic re-entrant structure.

\section{MECHANICAL PROPERTIES OF THE AUXETIC HONEYCOMBS}

Three auxetic honeycomb designs were presented in the current work namely, classical re-entrant, splined-reentrant and stiffened reentrant as shown in Fig.1. Analytical expressions of the mechanical properties of the classical reentrant honeycombs are presented in Table 1. Other designs were modelled using finite elements method to evaluate the mechanical properties. The material properties used in evaluating the mechanical properties of the honeycombs are shown in Table 2. The two new designs found to have better in-plane mechanical properties in $x$ and $y$ directions and still have the auxetic behavior in place as described in a previous work by the authors [19]. A comparison between the experimental and finite elements results presented in Table 3, which showed good agreement and showed the significant improvement of the in-plane properties.

\section{FREE VIBRATION ANALYSIS}

The vibration analysis for the three structures was conducted using the finite element method using ANSYS software. Finite element models were constructed using SHELL99 element in, each model was constructed using at least ( $3^{\star} 2$ to $\left.9^{\star} 8\right)$ unit cell. The displacements (translation and rotation) of all the nodes along the bottom edge of typically $4 \times 3$ unit cells were constrained to zero in both the $x, y$ and $z$ directions. The impulse force applied on the top edge at single node, which located in middle of top edge to simulate the free vibration of cantilever plate. Modal analysis is used to determine the natural frequencies and mode shapes of a structure in the ANSYS program. The natural frequencies and mode shapes are of important parameters in the design of a structure for dynamic loading conditions. The modal analysis in the ANSYS family of products is of linear analysis. Any nonlinearity such as plasticity and contact (gap) elements are ignored even though they are defined. Choose the mode from several mode-extraction methods; Block lanczos, supernode, PCG lanczos, reduced, asymmetric, damped and QR damped. Block lanczos is used in the current analysis to obtain mode shapes and corresponding natural frequencies for the two models. In the following section the effect of rib length on the natural frequencies of the honeycombs is explained.

\section{Effects of Rib Length on Natural Frequencies}

The natural frequency of any structure depends on its stiffness and mass. The current analysis studies the effect of rib length on natural frequency. The natural frequency values for the structure with $L=60 \mathrm{~mm}$ are consistently lower than the other structures in case of reentrant, splined and stiffened structures. Figure 2 shows the changes of the first natural frequencies of classic, splined and stiffened reentrant 
Table 1. Unitcell shape and expressions for the in-plane properties of the re-entrant hexagonal honeycombs.

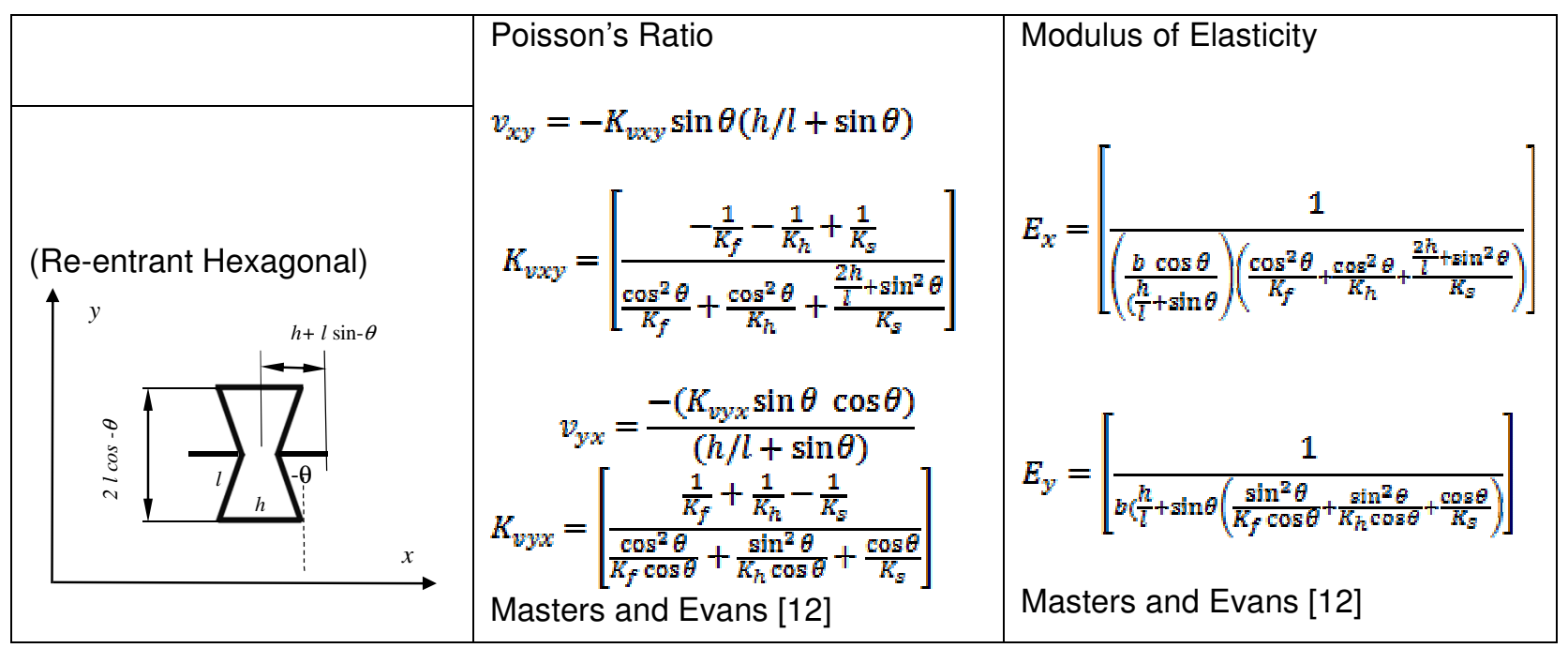

where $b$ is the depth of the topology and $K_{l}=E_{s} b(t / l)^{3}, K_{h}=\left[E_{s} b / 2\left(1+v_{s}\right)\right](t / l), K_{s}=E_{s} b(t / l)$

Table 2. Material and initial geometrical parameters of honeycomb modeled with different design [19].

\begin{tabular}{|c|c|c|c|}
\hline Parameters & Classical & Splined & Stiffened \\
\hline$h=I(\mathrm{~mm})$ & 42 & 42 & 42 \\
\hline$t_{h}=t_{l}(\mathrm{~mm})$ & 2.86 & 2.86 & 2.86 \\
\hline$\theta($ degree $)$ & \multicolumn{3}{|l|}{} \\
\hline$b(\mathrm{~mm})$ & 7.75 & 670 & 7.75 \\
\hline$h_{s}(\mathrm{~mm})$ & ---- & 20.45 & 20.45 \\
\hline$h_{l}(\mathrm{~mm})$ & ---- & ----- & 38 \\
\hline$R(\mathrm{~mm})$ & 0 & 44.5 & 44.5 \\
\hline$R_{l}$ & --- & ---- & 94.5 \\
\hline$E_{\text {rib }}(\mathrm{GPa})$ & Modulus of elasticity of steel $E_{\text {steel }}=210 \mathrm{MPa}$ \\
\hline$v_{\text {rib }}$ & \multicolumn{2}{|c|}{ Poisson's ratio of steel $v_{\text {steel }}=0.3$} \\
\hline
\end{tabular}

Table 3. Modulus of elasticity and Poisson's ratio versus different new designs of re-entrant honeycomb [19].

\begin{tabular}{|c|c|c|c|c|}
\hline \multicolumn{2}{|c|}{ Parameters } & Classical & Splined & Stiffened \\
\hline \multirow{2}{*}{$\begin{array}{c}\text { Modulus of } \\
\text { elasticity } E_{x T} \\
(M M P a)\end{array}$} & FEM (ANSYS) & 42.4 & 55.23 & 725.54 \\
\cline { 2 - 5 } & Experimental & 44.829 & 53.97 & 715.12 \\
\hline $\begin{array}{c}\text { Modulus of } \\
\text { elasticity } E_{y L} \\
(M P a)\end{array}$ & FEM (ANSYS) & 618.54 & 471.43 & 1343.93 \\
\cline { 2 - 5 } & Experimental & 623.8 & 467.67 & 1357.62 \\
\hline \multirow{2}{*}{ Poisson's ratio $v_{y x}$} & FEM (ANSYS) & -3.23 & -2.34 & -0.53 \\
\cline { 2 - 5 } & Experimental & -3.59 & -2.65 & -0.61 \\
\hline \multirow{2}{*}{ Poisson's ratio $v_{x y}$} & FEM (ANSYS) & -0.39 & -0.45 & -1.06 \\
\cline { 2 - 5 } & Experimental & -0.32 & -0.39 & -0.999 \\
\hline
\end{tabular}


honeycomb structure with rib length. Considering other parameters are constant, the first natural frequency gradually decreases with increased rib length because the stiffness of the element decreases due to the stiffness inversely proportional to the cubic length. The first longitudinal and twisting natural frequency about $y$-axis as shown in Figs. $3 a$ and $3 b$ have the same trend.

\section{MODE SHAPES OF REENTRANT HONEYCOMB STRUCTURES}

There are four types of vibration mode shapes observed in the different honeycomb structures: bending about $z$ axis, bending about $x$ axis, twisting about $y$ axis and longitudinal in $y$ axis. The bending modes of vibration (about $z$ axis) are observed at lower natural frequencies $(\sim 1 \mathrm{~Hz}$ to $\sim 1350 \mathrm{~Hz})$, whereas the longitudinal modes of vibration occurs (in y axis) at higher natural frequencies. Figs. 4 to 9 show the flexural and twisting modes of vibration respectively for different structures of reentrant honeycomb core.

\section{EXPERIMENTAL WORK}

The setup of vibration measurements for determination of the natural frequencies is shown in Fig. 6 and Fig. 7. The responses are shown in Fig. 8, which have been used to evaluate the different modes and vibration responses.

\section{RESULTS AND DISCUSSION}

The study was carried out on the classical re-entrant honeycomb structure and two other new modified versions of the honeycomb namely splined re-entrant and stiffened re-entrant. The proposed two new designs (splined and stiffened) reentrant honeycomb showed a significant improvement of in-plane stiffness of the honeycomb cores and keeping the auxetic behavior in action. However, the value of the Poisson's ratios decrease as the in-plan stiffness increases which shows that the reciprocal theory is applicable for auxetic honeycomb. Moreover, the study on varying the structures unit cell rib length carried out on the three structures showed that there is a significant effect of the rib length on the in-plane mechanical properties and natural frequencies of the structures.

Table 4 shows a comparison between experimental and finite element results for the first three natural frequencies for bending about $z$-axis, bending about $x$-axis, twisting and longitudinal modes for different classic, splined and stiffened reentrant. The different modes can be separated in ANSYS program by estimate the nodal displacement in all directions and a comparison between them, the selected mode type depending on the maximum nodal displacement. Experimental measurements for different modes depending on the position of accelerometer and hammer excitation point.

The natural frequencies for splined and stiffened reentrant found to be decreased when compared with classical reentrant for out-of-plane mode this can be referred to the increase of the overall mass. However, it increases for the in-plane modes because of the increase of structure stiffness. 
Table 4. Comparison of the first three natural frequencies for different modes and honeycombs.

\begin{tabular}{|c|c|c|c|c|c|c|c|c|c|c|c|c|c|}
\hline \multirow[b]{2}{*}{ Honeycomb } & \multirow{2}{*}{$\begin{array}{l}\text { Mode } \\
\text { Method }\end{array}$} & \multicolumn{3}{|c|}{ Bending about $z$} & \multicolumn{3}{|c|}{ Bending bout $x$} & \multicolumn{3}{|c|}{ Twisting about y } & \multicolumn{3}{|c|}{ Longitudinal along y } \\
\hline & & $\omega 1$ & $\omega 2$ & $\omega 3$ & $\omega 1$ & $\omega 2$ & $\omega 3$ & $\omega 1$ & $\omega 2$ & $\omega 3$ & $\omega 1$ & $\omega 2$ & $\omega 3$ \\
\hline \multirow{2}{*}{$\begin{array}{l}\text { Classic } \\
\text { reentrant }\end{array}$} & FE (ANSYS) & 75 & 532 & 1375 & 108 & 236 & 559 & 120.19 & 390.73 & 764.22 & 365.89 & 515.7 & 568.21 \\
\hline & experimental & 72 & 528 & 1388 & 108 & 225 & 539 & 121.7 & 391.25 & 766.5 & 367.5 & 520.4 & 571.2 \\
\hline \multirow{2}{*}{$\begin{array}{l}\text { Splined } \\
\text { reentrant }\end{array}$} & FE (ANSYS) & 48.5 & 323.5 & 923.5 & 80.5 & 199.7 & 476.5 & 120.36 & 369.77 & 723.63 & 398.25 & 505.77 & 569.81 \\
\hline & experimental & 49.44 & 334.8 & 930 & 87.89 & 197.1 & 470.4 & 118.6 & 368.4 & 726.8 & 392.6 & 509.44 & 571.32 \\
\hline \multirow{2}{*}{$\begin{array}{l}\text { Stiffened } \\
\text { reentrant }\end{array}$} & FE (ANSYS) & 40.5 & 274.5 & 883 & 110.5 & 465 & 1128 & 215.81 & 657.55 & 1226.4 & 1055.9 & 1329.9 & 1556.1 \\
\hline & experimental & 41.35 & 277.3 & 890 & 109.5 & 469 & 1130 & 211.5 & 663.3 & 1231 & 1060 & 1331.8 & 1560 \\
\hline
\end{tabular}

\section{CONCLUSIONS}

In this study, the vibrational characteristics of auxetic honeycomb cores were investigated. The study included theoretical analyses and experimental measurements of the honeycomb dynamic behaviors. A study on the effect of the honeycomb properties on the vibrational behaviors was conducted. Finite elements method was mainly employed for situations where the analytical models were not available Experimental evaluation of the structures properties were conducted to verify the analytical and the numerical results. Free vibration tests were carried on honeycomb samples made of steel using the laser cutting technique with different geometrical parameters. It is concluded that, the increase of the rib length reduces the natural frequencies of all structures under study. The classic reentrant honeycomb structures have high values of bending (out-off-plane) natural frequencies compared with splined and stiffened reentrant honeycomb this is referred to the effect of density of structures. The classical reentrant honeycomb structures have lower values of in-plane natural frequencies compared to the splined and stiffened reentrant honeycomb> this can be attributed to the effect of stiffness of the structures.

\section{REFERENCES}

[1] Bitzer, T. Honeycomb Technology: Materials, Design, Manufacturing, Applications and Testing, London, (1997).: Chapman \& Hall.

[2] A. Alderson, K. L Alderson, 2007. „Auxetic materials“ Proc. ImechE, 221 Part G ; 1 Aerospace Engineering, pp565-575.

[3] Gibson LJ, Ashby MF. Cellular solids, structure and properties. $2^{\text {nd }}$ ed. Cambridge: Cambridge University Press; 1997.

[4] D, Lakes R. Properties of a chiral honeycomb with Poisson's ratio -1. Int. J. Mech. Sci. 1996;39:305-314.

[5] Lakes R S, 1987, Foam structures with a negative Poisson's ratio Science 253 1038-40.

[6] Evans K 1991 The design of doubly curved sandwich panels with honeycomb cores Compos. Struct. 17 95-111. 
[7] A. Lorato, P. Innocenti, F. Scarpa, N. Ravirala, K M Zied, A. Alderson, K. Alderson, W Miller, C. W Smith, K. E Evans. "The transverse elastic properties of chiral honeycombs". Journal of Composites Science and Technology. doi:10.1016/j.compscitech.2009.07.008.

[8] A. Alderson, K. L Alderson, D. Attard, K. E Evans, P. S Farrugia, R. Gatt, J. N Grima W. Miller, N. Ravirala, C. W Smith, K. M Zied. 2009."Elastic constants of 3-, 4- and 6-connected chiral and anti-chiral honeycombs subject to uniaxial in-plane loading". Journal of Composites Science and Technology. doi:10.1016/j.compscitech.2009.07.009.

[9] A. Alderson, K. L. Alderson, N. Ravirala, K.M. Zied. The in-plane linear elastic constants and out of plane bending of 3-coordinated ligament and cylinderligament honeycombs" Journal of Composites Science and Technology. doi:10.1016/j.compscitech.2009.07.010.

[10] J. Whitty, F. Nazare, A. Alderson. "Modelling the effect of density variations on the in-plane Poisson's ratios and Young's modui of periodic conventional and re-entrant honeycombs- Part 1: rib thickness variation." Cellular Polymers 21(2), pp69-97, 2005.

[11] Evans K E 1991 Auxetic polymers: a new range of materials Endeav. New Ser. 15 170-4

[12] Masters I and Evans K 1996 Models for the elastic deformation of honeycombs Compos. Struct. 35 403-22

[13] C.I. Thill, J. Etches, I. Bond, K. Potter, and P. Weaver, 2008, "Morphing Skins," The Aeronautical Journal, No. 3216.

[14] Martin J, Heyder-Bruckner J J, Remillat C, Scarpa F, Potter K and Ruzzene M 2008 The hexachiral prismatic wingbox concept Phys. Status Solidi b 245 $570-7$

[15] Spadoni A, Ruzzene M and Scarpa F 2006 Dynamic response of chiral trusscore assemblies J. Intell. Mater. Syst. Struct. 17 941-52

[16] Bornengo D, Scarpa F and Remillat C 2005 Evaluation of hexagonal chiral structure for morphing airfoil concept Proc. Inst. Mech. Eng. G 219 185-92

[17] Scarpa F, Smith F C, Chambers B and Burriesci G 2003 Mechanical and electromagnetic behaviour of auxetic honeycomb structures Aeronaut. J. 107 $175-83$.

[18] Whitty J.P.M., Alderson A., Myler P., Kandola B., Towards the design of sandwich panel composites with enhanced mechanical and thermal properties by variation of the in-plane Poisson's ratios. Composites. Part :Appl. Science and Manufacturing, 2003, 34, 525-534.

[19] K. Zied, M. Osman, and T. Elmahdy, 2015, "Enhancement of the in-plane stiffness of the hexagonal re-entrant auxetic honeycomb cores" Phys. Status Solidi B, 1-8 (2015) / DOI 10.1002/pssb.201552164 


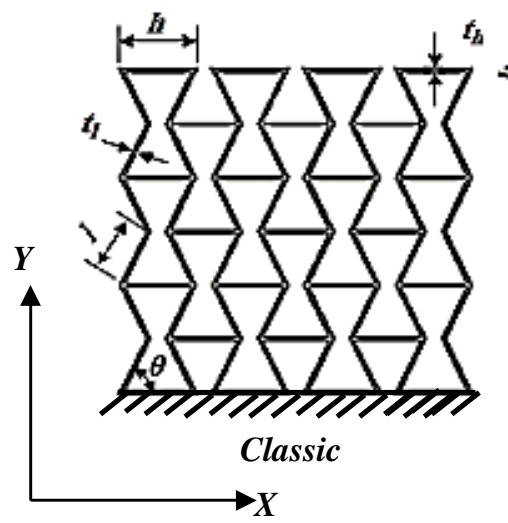

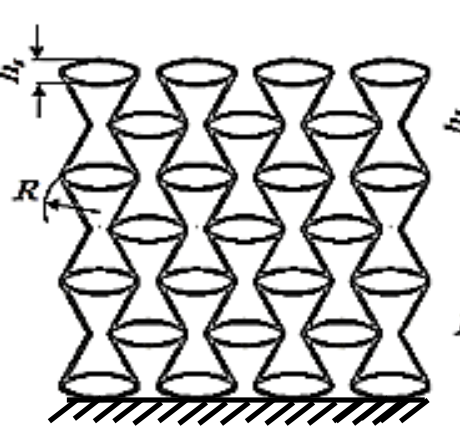

Spline

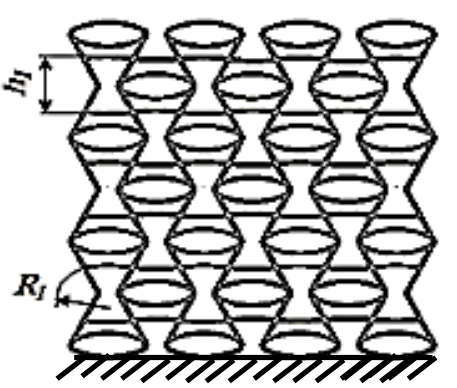

Stiffened

Fig. 1. Classic, splined and stiffened re-entrant honeycomb geometrical parameters and co-ordinate system [19].

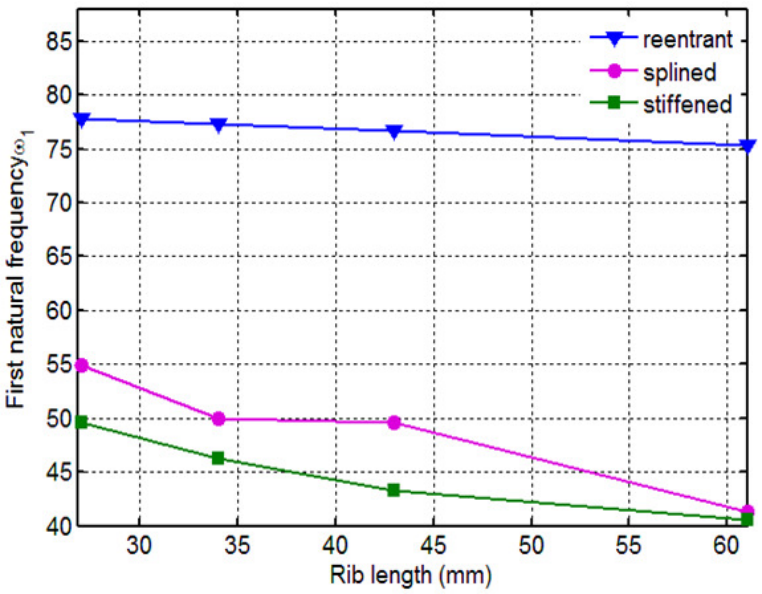

(a)

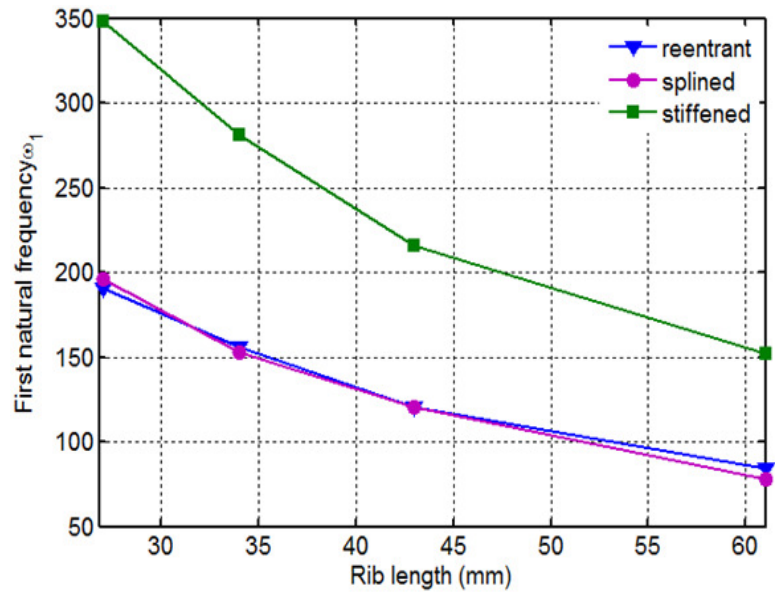

(b)

Fig. 2. Effect of rib length on the first bending natural frequency for different honeycomb about (a) $z$ axis (b) $x$ axis.

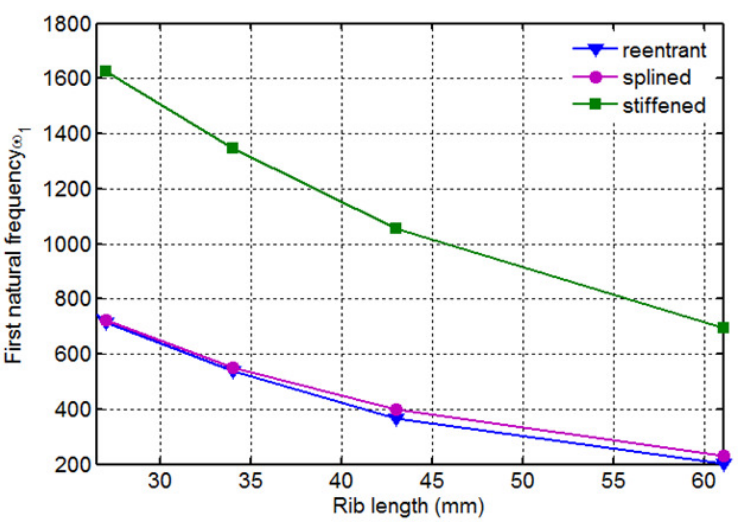

(a)

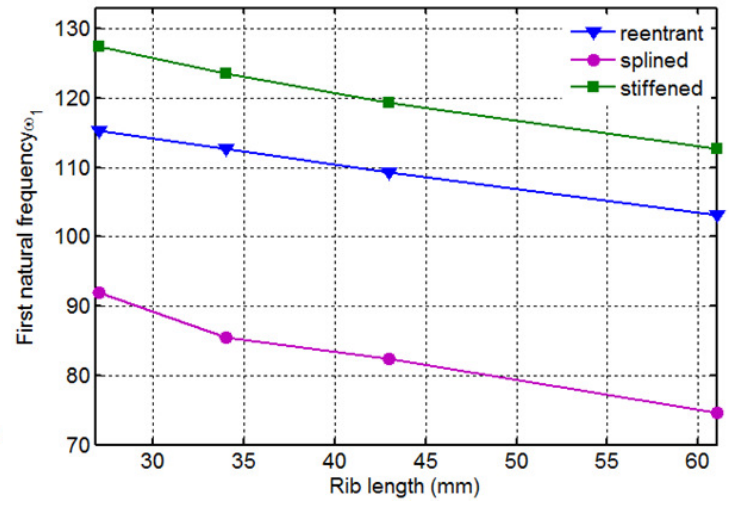

(b)

Fig. 3. Effect of rib length on the first natural frequency for different honeycomb in y axis (a) longitudinal (b) Twisting. 
The First Mode

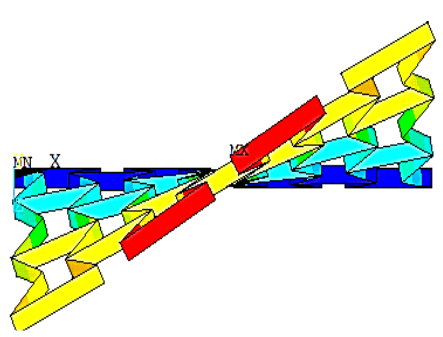

The Second Mode

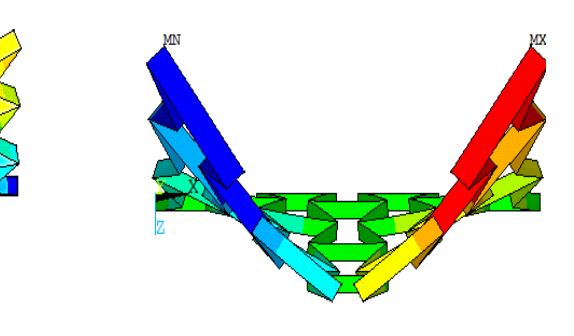

The Third Mode

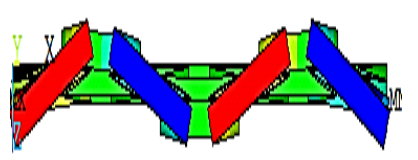

(a)
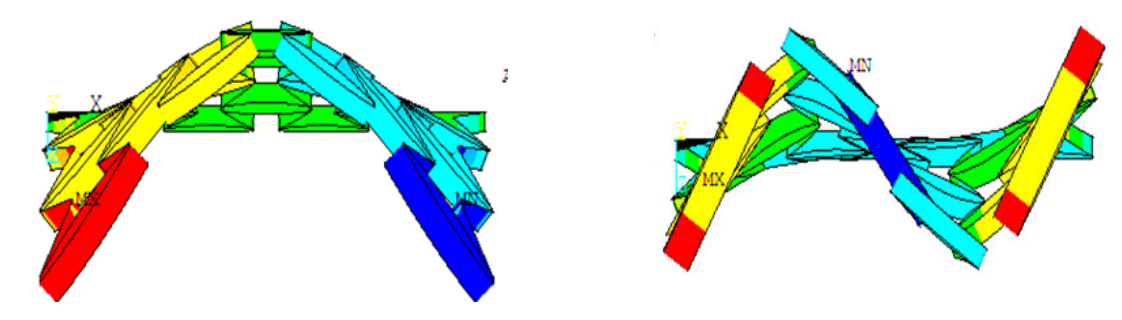

(b)

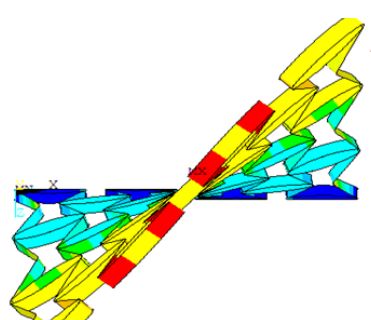

(c)
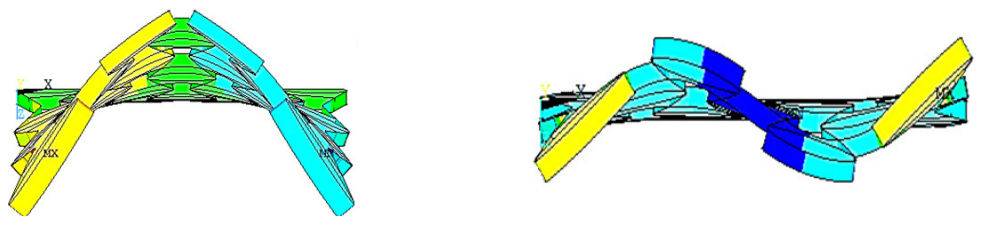

Fig. 4. The first three natural twisting modes of stiffened reentrant: ((a) Classic re-entrant (b) splined reentrant (c) Stiffened reentrant. 
The First Mode

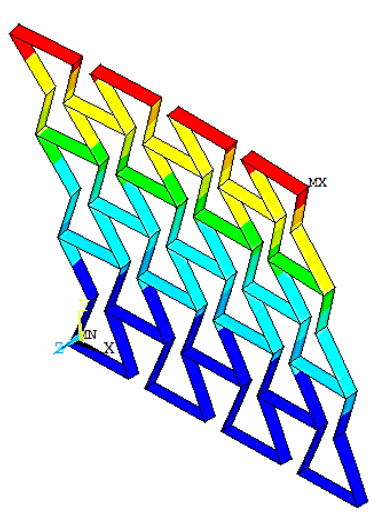

The second Mode
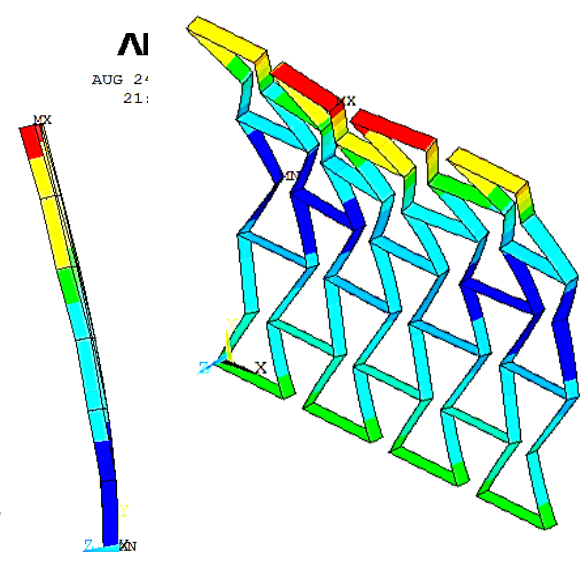

(a)
The third Mode
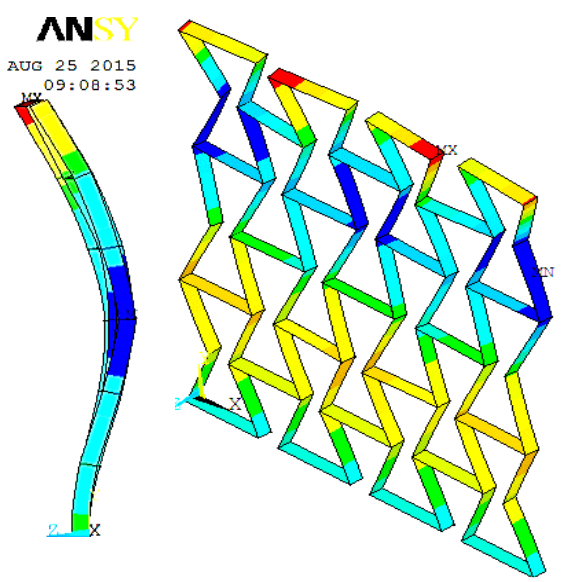

$\mathbf{N N}$

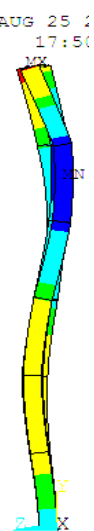

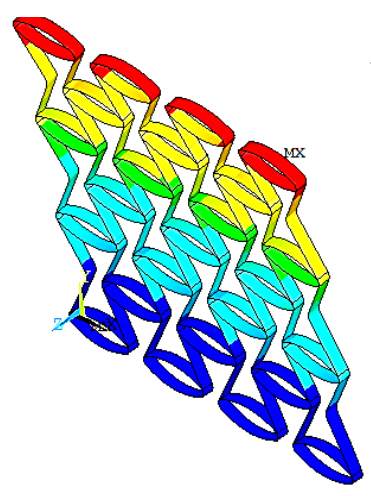
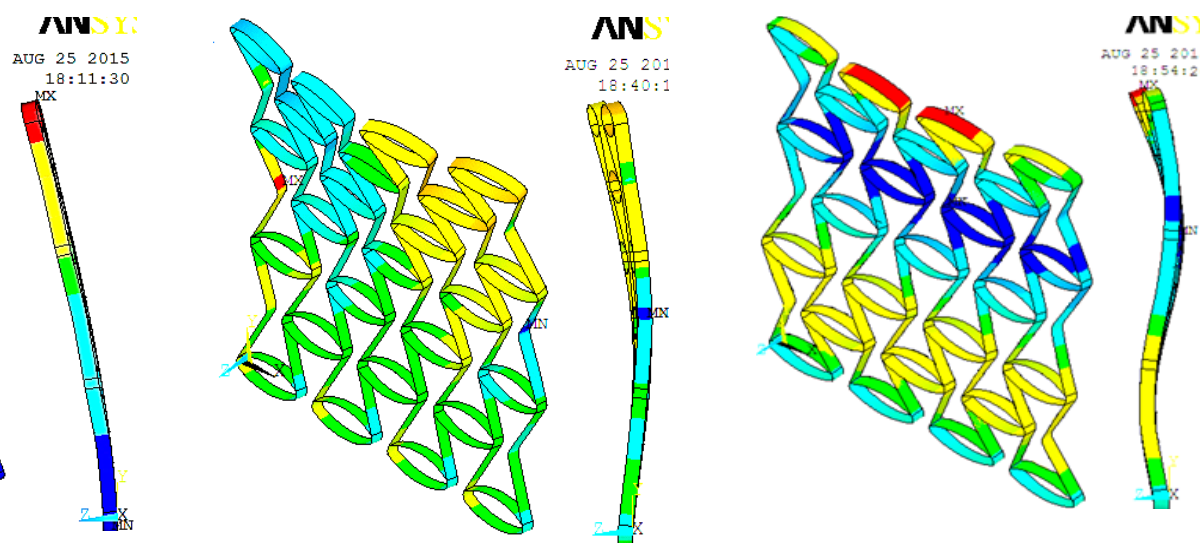

(b)
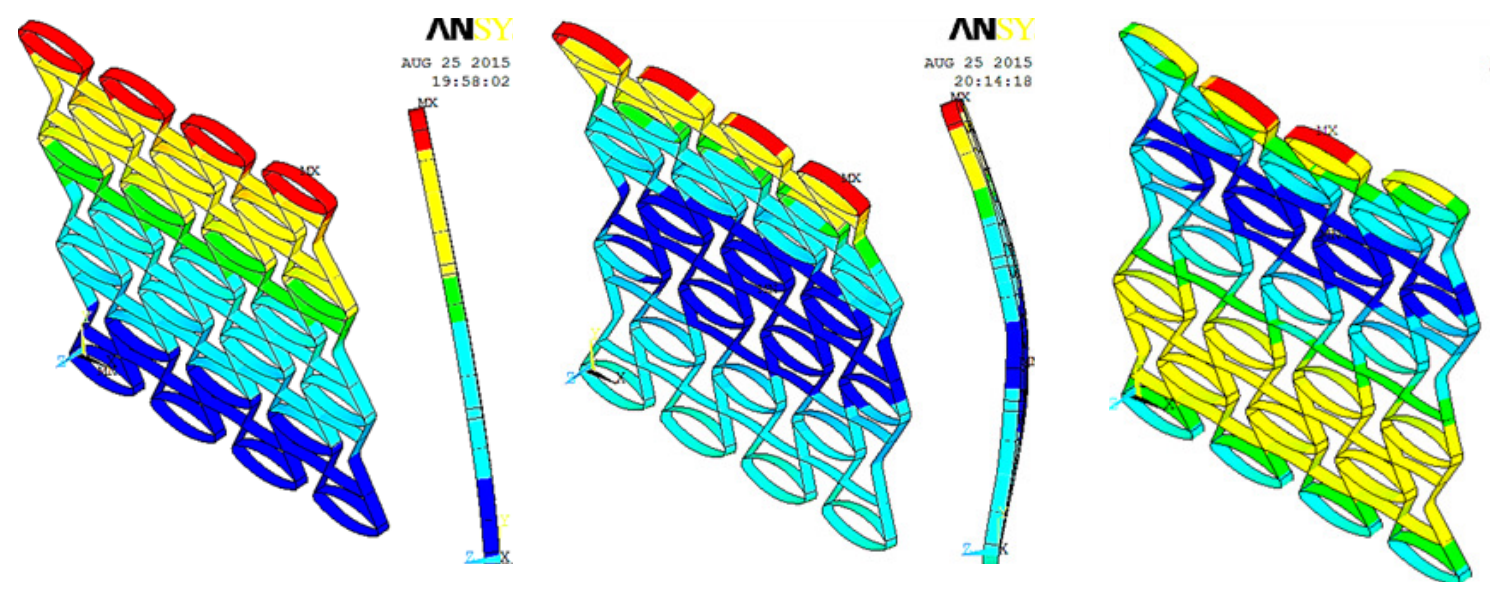

$\mathbf{N N}$

(c)

Fig. 5. The first three natural bending modes of: (a) Classic re-entrant, (b) splined reentrant, (c) Stiffened reentrant. 


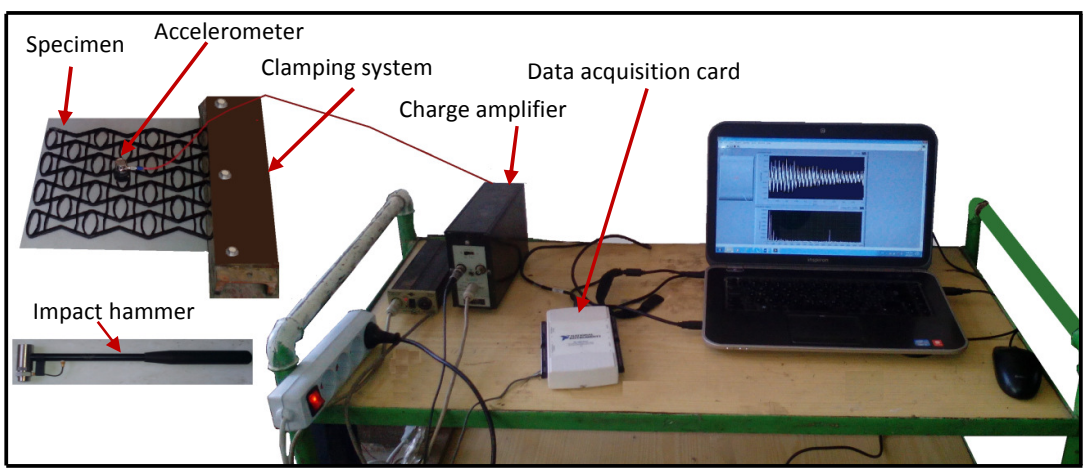

Fig. 6. Experimental setup.
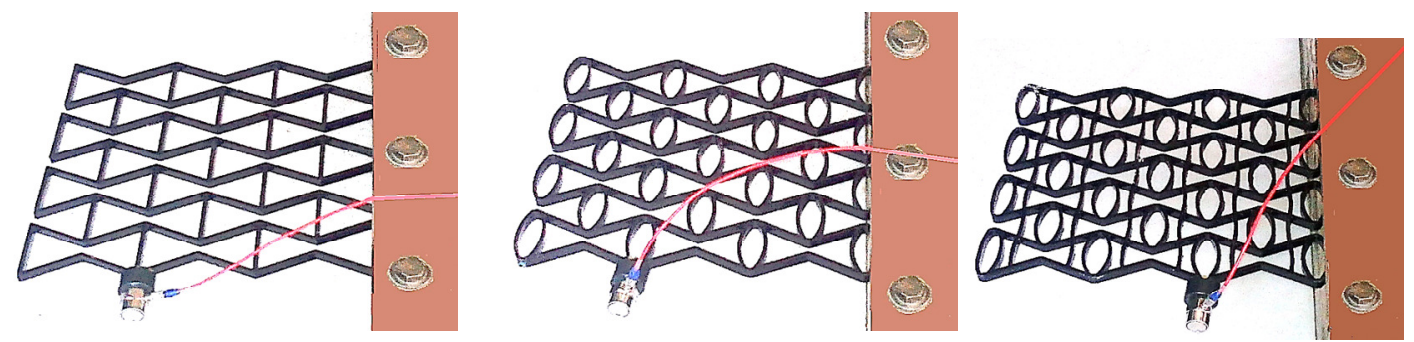

Fig. 7. Test samples during measuring the bending mode about $z$ axis and twisting mode about y axis.

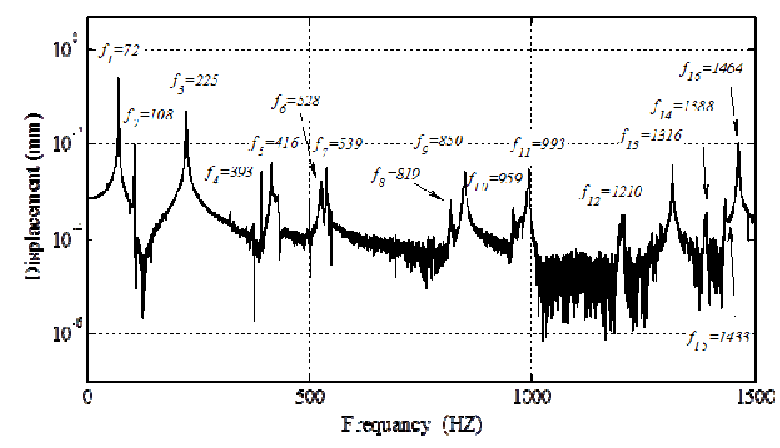

(a)

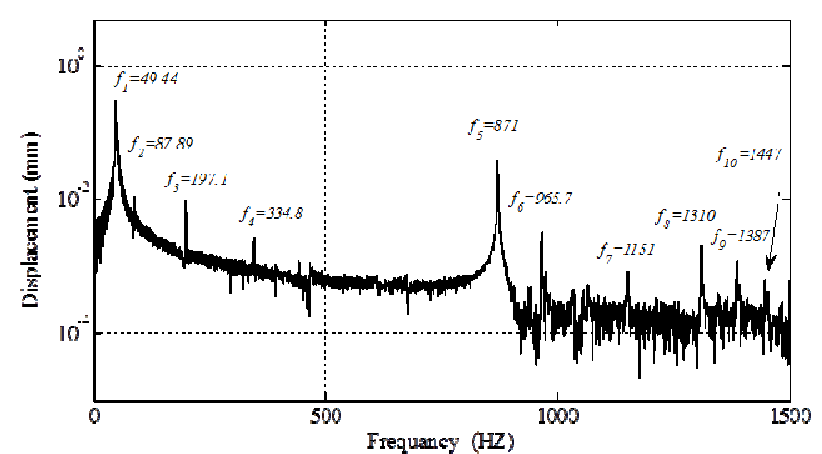

(b)

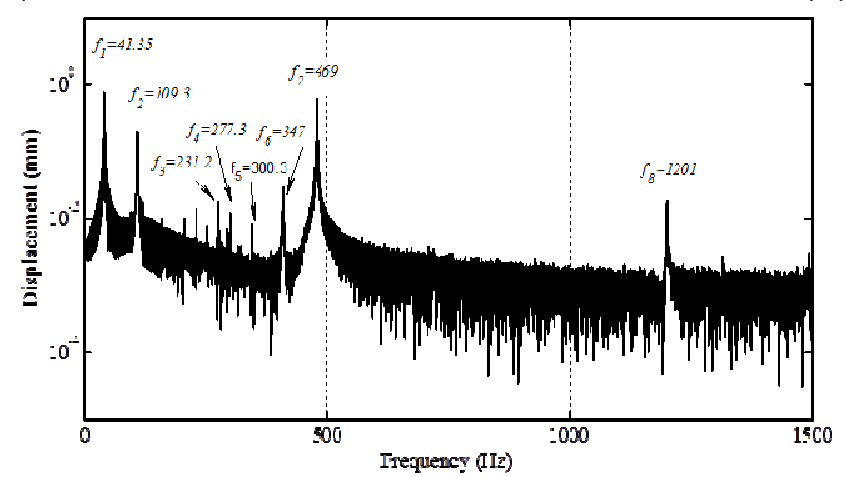

(c)

Fig. 8. Experimental data for (a) Reentrant honeycomb, (b) splined reentrant honeycomb (c) Stiffened reentrant honeycomb. 